\title{
Spatiotemporal coding of inputs for a system of globally coupled phase oscillators
}

\author{
John Wordsworth and Peter Ashwin \\ Mathematics Research Institute, School of Engineering, Computing and Mathematics, University of Exeter, Exeter, \\ EX4 4QF, United Kingdom
}

(Received 3 June 2008; published 4 December 2008)

\begin{abstract}
We investigate the spatiotemporal coding of low amplitude inputs to a simple system of globally coupled phase oscillators with coupling function $g(\phi)=-\sin (\phi+\alpha)+r \sin (2 \phi+\beta)$ that has robust heteroclinic cycles (slow switching between cluster states). The inputs correspond to detuning of the oscillators. It was recently noted that globally coupled phase oscillators can encode their frequencies in the form of spatiotemporal codes of a sequence of cluster states [P. Ashwin, G. Orosz, J. Wordsworth, and S. Townley, SIAM J. Appl. Dyn. Syst. 6, 728 (2007)]. Concentrating on the case of $N=5$ oscillators we show in detail how the spatiotemporal coding can be used to resolve all of the information that relates the individual inputs to each other, providing that a long enough time series is considered. We investigate robustness to the addition of noise and find a remarkable stability, especially of the temporal coding, to the addition of noise even for noise of a comparable magnitude to the inputs.
\end{abstract}

DOI: 10.1103/PhysRevE.78.066203

PACS number(s): 05.45.Xt, 87.18.Hf

\section{INTRODUCTION}

Coupled phase oscillators provide a simple mechanism for modeling collective activity such as synchronization between clusters of neurons (see, for example, [1]). By taking the simplest dynamical model of an oscillator, they give tractable models for neural systems that can be derived rigorously in the limit of weak coupling, and which give insight into a very wide range of neural applications.

This paper investigates a simple system of this form with mean field (global) coupling of nearly identical phase oscillators, through a coupling function that robustly gives slow switching [2] in the case of identical oscillators-the "ideal" system. The dynamics of the ideal system consists of attractors that are robust heteroclinic attractors between cluster states, robust to perturbations in the coupling function [3].

The dynamics of heteroclinic networks gives examples of winnerless competition models that are proposed to be good models for certain neural systems [4,5], in particular, those that involve conversion of a steady multidimensional input into a persistent spatiotemporal code. This motivates the current paper; we investigate how small inputs to the system of coupled oscillators [6] result in a spatiotemporal coding of those inputs. The inputs are implemented as perturbations to the natural frequencies of the oscillators. The coding can be used to classify the input ordering (via the spatial coding, i.e., the ordering of visits to a number of cluster states) and the input magnitudes (via the temporal coding, namely, the residence times at each of the cluster states). We completely characterize the spatial coding in terms of its combinatorics and successfully predict magnitudes of inputs via the temporal coding. We also investigate the influence of noise on the resulting code and show (a) that the spatial coding is very resistant to noise as long as it has a lower magnitude than the inputs, and (b) that the entropy of the spatial coding increases in a very sharp manner as the noise magnitude increases beyond the input magnitude.

We consider a set of globally coupled phase oscillators of the form

$$
\dot{\theta}_{n}=\omega_{n}+\frac{1}{N} \sum_{m=1}^{N} g\left(\theta_{n}-\theta_{m}\right)+\eta \omega_{n}(t), \quad n=1, \ldots, N,
$$

where $\theta_{n}$ is the phase of the $n$th oscillator such that $\theta_{n}$ $\in[0,2 \pi)$ and $g(\phi+2 \pi)=g(\phi)$ for all $\phi \in R$. $\omega_{n}$ is the natural frequency of the $n$th oscillator, and $w_{n}(t)$ is uncorrelated white noise such that the associated random walk has a unit growth of variance per unit time. The quantity $\eta$ is the noise strength and we include independent and identically distributed (i.i.d.) additive noise independently to each phase, with zero mean, such that the standard deviation scales as $\eta$ per unit time.

We will restrict our discussion to coupling of the form

$$
g(x)=-\sin (x+\alpha)+r \sin (2 x+\beta),
$$

where $\alpha=1.7, \beta=-2.0$, and $r=0.2$ [6], although qualitatively the dynamics are similar for an open set of nearby parameter values.

The particular form (2) includes all Fourier components up to the second harmonic and includes dynamics that are not robustly observable for fewer than this number of components. The coupling (1) was investigated in [6] where it was shown that for a system of five oscillators the only attractors for this coupling involve switching between a total of 30 different periodic orbits that have a clustering into two pairs of two oscillators and a singleton; we describe this as $(2,1,2)$ clustering. More generally, in systems of $2 k+1$ globally coupled phase oscillators, this coupling leads to dynamics in which the model becomes partially synchronized with oscillators in $(k, 1, k)$ clusters. These attractors consist of $\frac{(2 k+1) !}{(k !)^{2}}$ unstable manifolds, each with $k$ branches leading to different, symmetrical unstable $(k, 1, k)$ clusters, forming a robust heteroclinic network.

The coupling (2) is chosen to give nontrivial dynamical clustering behavior that is robust for such systems. The case $\beta=0$ has been studied by others $[7,8,2,3]$ and also exhibits robust heteroclinic attractors, although between two-cluster 
TABLE I. An enumeration of the set of possible $(2,1,2)$ cluster states for a fully symmetrical system with $N=5$ oscillators. The state $s_{1}=S_{33211}$ corresponds to the phases clustered as $\left(\phi_{1}+\xi, \phi_{1}+\xi, \phi_{2}+\xi, \phi_{3}+\xi, \phi_{3}\right.$ $+\xi)$.

\begin{tabular}{lllll}
\hline \hline$s_{1}=S_{33211}$ & $s_{2}=S_{11233}$ & $s_{3}=S_{31231}$ & $s_{4}=S_{13213}$ & $s_{5}=S_{31213}$ \\
$s_{6}=S_{13231}$ & $s_{7}=S_{13321}$ & $s_{8}=S_{31123}$ & $s_{9}=S_{13123}$ & $s_{10}=S_{31321}$ \\
$s_{11}=S_{33121}$ & $s_{12}=S_{11323}$ & $s_{13}=S_{11332}$ & $s_{14}=S_{33112}$ & $s_{15}=S_{31312}$ \\
$s_{16}=S_{13132}$ & $s_{17}=S_{13312}$ & $s_{18}=S_{31132}$ & $s_{19}=S_{21133}$ & $s_{20}=S_{23311}$ \\
$s_{21}=S_{23131}$ & $s_{22}=S_{21313}$ & $s_{23}=S_{21331}$ & $s_{24}=S_{23113}$ & $s_{25}=S_{32113}$ \\
$s_{26}=S_{12331}$ & $s_{27}=S_{12313}$ & $s_{28}=S_{32131}$ & $s_{29}=S_{12133}$ & $s_{30}=S_{32311}$ \\
\hline \hline
\end{tabular}

states rather than three-cluster states. The precise dynamics that we consider is described in detail in the following section.

In Sec. II we examine the robust clustering dynamics of the proposed model in the ideal system of identical oscillators. We then investigate the effect of applying fixed inputs to the system by detuning individual oscillators. Inputs of this fashion force the clustering to change in a predictable manner as the system explores part of the heteroclinic network, such that one can completely predict the sequence of states that a given trajectory will pass through. There is a predictable relationship between the relative strength of the inputs and the time spent near specific cluster states. In this section we describe a method of interpreting output from our proposed model as a sequence of codes that represent the spatial properties of each cluster that the system spends time near (spatial coding) as well as a sequence representing the amount of time the system spends near each state (temporal coding). Combining these codes generates a spatiotemporal coding for the system that can be used to completely resolve information about relative differences between inputs.

In the case where the strength of each input is fixed and unequal the branch followed when leaving a cluster state is predetermined. In Sec. III we investigate this case where the network collapses to a network of two individual components, which both result in a periodic cycle of cluster states of length 6 . After discussing this theory we justify our work by reference to numerical simulations. In Sec. III C we explore the inverse problem-determining the relative strengths of the inputs to the system from a given length of spatiotemporal coding. For an unknown uniform detuning we show how one can exactly determine the detuning by examining the spatiotemporal code.

In many neural systems noise plays an important role. In Sec. IV we consider the entropy of code generated by our model in the presence of both uniform detuning and random inputs in the form of noise. We observe that the system is highly stable in the presence of noise up to a magnitude equal to the magnitude of the inputs and characterize the amount of information from the spatial coding that is lost (entropy) due to the noise present in the system.

Finally, in Sec. V we briefly explore the dynamics for situations where there are $N>5$ oscillators. While there are many similarities to the simple case of $N=5$ oscillators, the network of cluster states grows exceedingly quickly, hence the problem becomes much more complex. We also discuss the surprising resilience that the proposed system shows to noise.

\section{SPATIOTEMPORAL CODING OF INPUTS}

We consider the smallest number of oscillators that can generate nontrivial robust switching between cluster state dynamics, namely, the case with five oscillators $(N=5, k=2)$ [6]. For $N=4$ oscillators there can be robust switching, but the network will be trivial [8].

\section{A. Network of cluster states}

For the fully symmetric case with no input, there is a uniform natural frequency across all of the oscillators $\left(\omega_{i}\right.$ $=\omega_{c}$ for $\left.n=1, \ldots, N\right)$ and no noise $(\eta=0)$. This case is described in [6] as having an attractor composed of 30 saddle periodic orbits (states) in phase space, each of which has a one-dimensional unstable manifold whose branches limit to two other symmetrically related states. For no input, the system will approach this network of states, progressively staying longer near each state. In the presence of noise $(\eta>0)$ the system will explore a random path on the network.

We refer to an l-cluster state of type $\left(N_{1}, \ldots, N_{t}\right)$ as a state where each oscillator has one of $l$ different relative phases $\phi_{1}, \ldots, \phi_{l}$, up to addition of the same constant to all phases. For each $r$ there are $N_{r}$ oscillators with phase $\phi_{r}$, up to addition of a constant, such that $\sum_{r=1}^{l} N_{r}=N$ [6]. For example, the oscillator phases of a $(2,1,2)$ cluster state are grouped into one of three values, of the form $\left(\phi_{1}, \phi_{1}, \phi_{2}, \phi_{3}, \phi_{3}\right)$ or some permutation thereof. For an examination of the existence and stability of general cluster states in such a system, see [9].

For the $(2,1,2)$ clusters we can enumerate all 30 permutations as the states $s_{k}, k=1, \ldots, 30$ listed in Table I. We write $s_{k}=S_{j_{1}^{k} \ldots j_{5}^{k}}$ to denote the state where the $l$ th oscillator is in the $j_{l}^{k}$ th cluster. For example, we say $\theta \in s_{1}=S_{11233}$ if and only if $\left(\theta_{1}, \ldots, \theta_{5}\right)=\left(\phi_{1}, \phi_{1}, \phi_{2}, \phi_{3}, \phi_{3}\right)+\xi(1,1,1,1,1)$ for some $\phi_{1}<\phi_{2}<\phi_{3}<\phi_{1}+2 \pi$.

As outlined in [6] each of the cluster states in Table I has a one-dimensional unstable manifold that connects to two other such states. We describe each close approach to a saddle cluster state as an epoch [10].

More precisely, if $s_{i}=S_{j_{1}^{i} \ldots j_{5}^{i}}$ and $s_{k}=S_{j_{1}^{k} \ldots j_{5}^{k}}$, then the unstable manifold of $s_{i}$ connects to the state $s_{k}$ on advancing the phase of the $p$ th oscillator by a small amount, precisely $O_{p}\left(s_{i}\right)=s_{k}$, where 


$$
O_{p}\left(s_{i}\right)=s_{k} \Leftrightarrow\left\{\begin{array}{ccc}
k=i & & \left(\text { if } j_{p}^{i} \neq 3\right) \\
j_{l}^{k}=3 & \text { if } \left.j_{l}^{i}=1 \quad \text { if } j_{p}^{i}=3\right) \\
j_{l}^{k}=1 & \text { if } \left.j_{l}^{i}=2 \quad \text { (if } j_{p}^{i}=3\right) \\
j_{l}^{k}=1 & \text { if } j_{l}^{i}=3 \text { and } l \neq p & \text { (if } \left.j_{p}^{i}=3\right) \\
j_{l}^{k}=2 & \text { if } j_{l}^{i}=3 \text { and } l=p & \left(\text { if } j_{p}^{i}=3\right) .
\end{array}\right.
$$

From the signs of the eigenvalues of the linearization we infer that perturbations that separate the oscillators in group 1 will decay over time. Consequently, we describe the cluster of oscillators in the group labeled by 1's as the stable cluster. Conversely, perturbations that separate the oscillators in group 3 will grow with time. For this reason, we describe the cluster of oscillators in group 3 as the unstable cluster. Investigating the dynamics around the other eigenvectors shows that there are no other perturbations that grow with time.

Using the function $O_{p}$ the dynamics on the network of cluster states can be represented as a directed graph, where each cluster state is represented by a node, and each branch of its unstable manifold is represented by a directed edge.

Considering both the information about $O_{p}$ and the states as in Table I, one can verify that the graph of cluster states collapses to two components, each of which has an eventual period 6 terminating cycle listed in Table I, as shown in [6].

\section{B. Response of the network to inputs (detuning)}

Let us write

$$
\omega_{n}=\omega_{0}+\delta_{n},
$$

where $\delta_{n} \ll 1$ and $\omega_{0}$ are constants; we can choose $\delta_{1}=0$ without loss of generality. Describe the amplitude of detuning for oscillator $n$ as $\delta_{n}$. We describe the response of the network to differences in $\omega_{n}$ (steady inputs) by using the operator $O_{p}$. In particular, for any given state $s_{i}$ we expect that $s_{k}$ follows $s_{i}$ precisely if $\omega_{p}>\omega_{q}$, where $p, q$ are the two oscillators in the unstable cluster and $O_{p}\left(s_{i}\right)=s_{k}$.

Suppose that the system is at, or near, a cluster state $s_{i}$ and its unstable cluster consists of the oscillators $p, q$. For convenience define the detuning in the unstable cluster to be

$$
\delta_{u}=\omega_{p}-\omega_{q} .
$$

If $\delta_{u}>0$ then the next expected state will be $O_{p}\left(s_{i}\right)$, while if $\delta_{u}<0$ the next expected state will be $O_{q}\left(s_{i}\right)$. Without loss of generality we can always assume that $p, q$ in the unstable cluster are chosen such that $\delta_{u} \geqslant 0$.

Moreover, we expect from [6,11] that the time of residence $\tau\left(s_{i}\right)$ near a given state $s_{i}$ is approximately given by

$$
\tau\left(s_{i}\right) \approx-\frac{1}{\lambda} \ln \left(\delta_{u}\right)+K
$$

at leading order $\delta_{u} \rightarrow 0$, where $\lambda>0$ is the unstable eigenvalue corresponding to the rate of growth of any perturbations that break the unstable cluster, and $K$ is a fixed time related to the minimum transition time along a connection between states.
Note that Eq. (5) implies that we can infer the detuning of the unstable cluster from the time of residence near that cluster

$$
\delta_{u} \approx e^{-\lambda(\tau-K)} .
$$

\section{Coding by sequence of states and residence times}

The sequence of cluster states, and the residence time near each cluster state, can be considered as a spatiotemporal coding sequence that represents the dynamical state of the system. We represent a trajectory near the heteroclinic network as a sequence that consists of a numeric index to reference the cluster state that the system was near during an epoch and the amount of time spent near that state-the residence time or the epoch length.

One can fairly easily determine whether a solution is near one of the cluster states in Table I. On a trajectory $\left(\theta_{1}(t), \ldots, \theta_{N}(t)\right)$ close to the network of cluster states $s_{i}$ we choose a tolerance $\xi$ such that $\left|s_{k}-s_{j}\right|>2 \xi$ for $k \neq j$ and then define the spatial state at time $t$ as follows:

$$
C(t)= \begin{cases}k: & \left|\theta(t)-s_{k}\right|<\xi \\ 0 & \text { otherwise. }\end{cases}
$$

i.e., $C(t)=k$ if and only if the trajectory is close to $s_{k}$ at time t. $C(t)=0$ implies that the trajectory is not near any classifiable state; this usually means that it is in transit between two such states [12]. We define an increasing sequence of times $t_{n}$ such that the $n$th epoch starts at time $t_{n}$ if the following holds:

$$
C\left(t_{n}\right) \neq 0,
$$

$$
\begin{gathered}
C(t) \neq C\left(t_{n}\right) \quad \text { for } t<t_{n} \quad \text { with } \quad\left|t_{n}-t\right| \text { arbitrarily small, } \\
C(t)=C\left(t_{n}\right) \quad \text { for } t_{n}<t<t_{n}+T_{\min },
\end{gathered}
$$

and there are no epochs between $t_{n-1}$ and $t_{n}$, where $T_{\min }>0$ is a fixed "minimum residence time." This enables us to classify a trajectory as a discrete time sequence of states $\left\{C_{n}\right\}$ associated with the $n$th epoch by

$$
C_{n}=C\left(t_{n}\right),
$$

where because of the instability of each state, $C_{n} \neq C_{n-1}$. This sequence is simply the measured itinerary of states and will depend on the precise initial conditions, the parameters, and any input (detuning) or noise added to the system. We call this the spatial coding sequence of the trajectory. Note that the structure of the network in Fig. 1 will limit the possible sequences that can appear as a spatial coding.

In addition to the spatial coding sequence of epochs we define a temporal coding as a sequence of residence times by

$$
T_{n}=\sup \left\{t>0: C\left(t_{n}\right)=C\left(t_{n}+s\right) \text { for all } 0<s<t\right\} .
$$

Note that $T_{n}>T_{\min }$ but the coding sequence is typically unaffected by $T_{\min }$ as the value of $T_{\min }$ is relatively small. $T_{n}$ is the length of the time interval for which $C(t)=C_{n}$ for a given $n$. The spatiotemporal code is then the sequence 


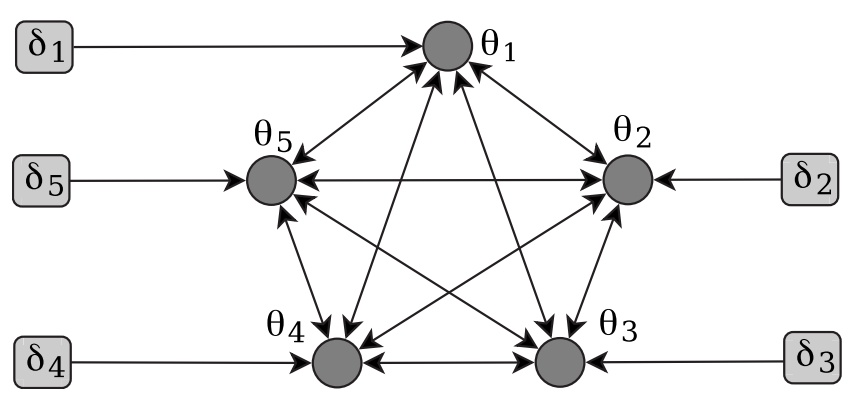

FIG. 1. A system of $N=5$ phase oscillators whose natural frequency is $\omega_{i}$ and phase is $\theta_{i}$. The oscillators are coupled with meanfield (global) coupling where each oscillator receives onedimensional input in the form of detuning its natural frequency. Each oscillator is coupled equally to all other oscillators by the coupling function $g(x)=-\sin (x+\alpha)+r \sin (2 x+\beta)$.

$$
\left\{\left(C_{n}, T_{n}\right): n=1,2,3, \ldots\right\} .
$$

An example showing a trajectory and its spatiotemporal code is illustrated in Fig. 2.

\section{DETUNING IN THE ABSENCE OF NOISE}

The dynamics of the case where noise or impulses drive the system has been explored in some detail [6]; the case where we apply a vector of constant inputs to the system, specifically, by altering the natural frequency of each oscillator by a fixed amount, deserves further investigation. We expand upon [6] by considering not only the spatial coding generated by such a system, but also the temporal coding.

\section{A. Uniform detuning}

The case of uniform detuning

$$
\omega_{n}=\omega_{0}+(n-1) \delta,
$$

where $\delta$ is constant, was briefly considered in [6]. By examining the ordering of the unstable clusters, each state will have a preferred direction of switching (4) determined by the choice of $p, q$ in the unstable cluster such that $\delta_{u}>0$. Moreover, uniform detuning implies that

$$
\delta_{u}=k \delta
$$

where $k \in\{1,2,3,4\}$. This means that each node on the graph shown in Fig. 3 has only one outgoing directed edge and so all directed paths on the graph become eventually periodic after a possible initial transient. In fact, as shown in [6], the graph reduces to two components each of which has an eventual period 6 coding sequence. Moreover, the residence times are determined by $\delta_{u}$ which, by Eq. (8) means that the residence times can be used to determine $\delta$.

Now consider the period 6 coding sequences that appear after the initial transient states have been passed in the presence of uniform detuning. We show how one can determine these sequences with a combinatorial method. After examining the possible sequences, one can verify that the only two periodic sequences for uniform detuning will be as listed in Table II.

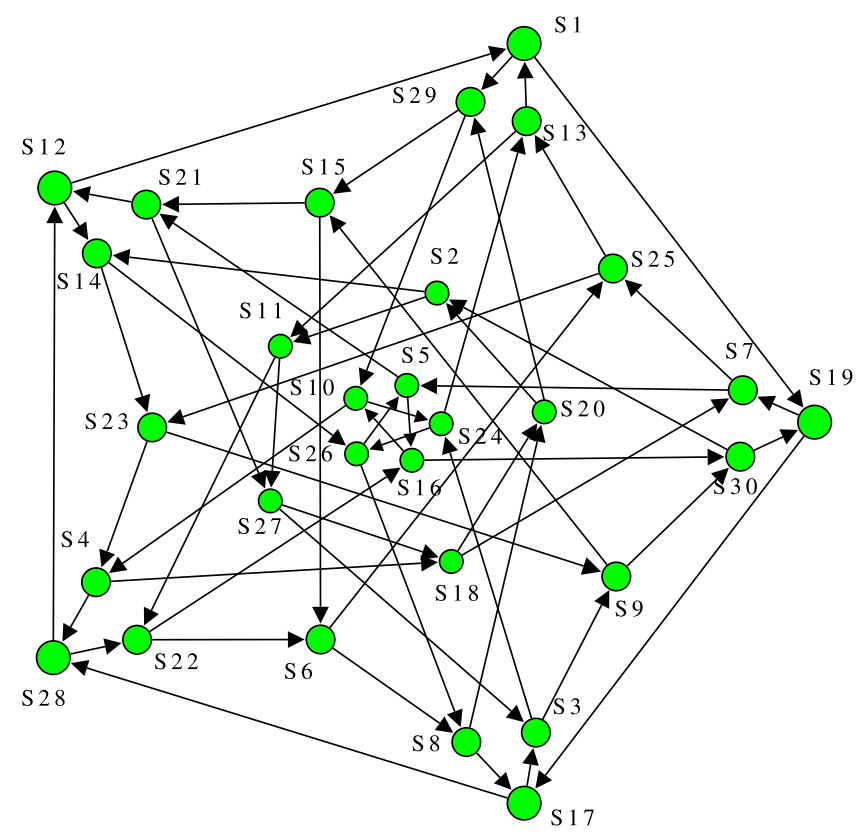

FIG. 2. (Color online) A graphical representation of the robust heteroclinic network generated by the system depicted in Fig. 1 where each node $s_{i}(i=1, \ldots, 30)$ represents a saddle point in phase space. Each directed edge leaving state $s_{i}$ represents an individual branch of the unstable manifold where differences in the frequencies will drive the system along a particular edge from a given node.

The period 6 sequences shown in Table II are generated for uniform detuning (8) such that $\omega_{1}<\omega_{2}<\omega_{3}<\omega_{4}<\omega_{5}$ but any such detuning sequence can be considered to have the same effect by permutation.
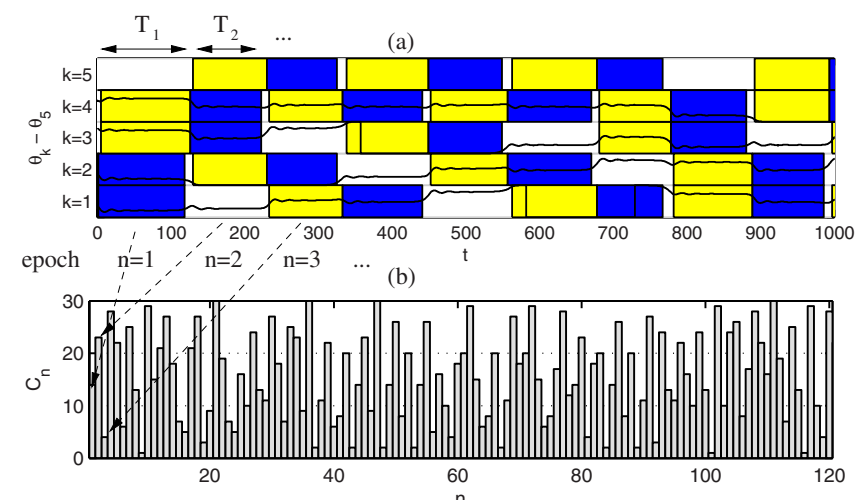

(c)

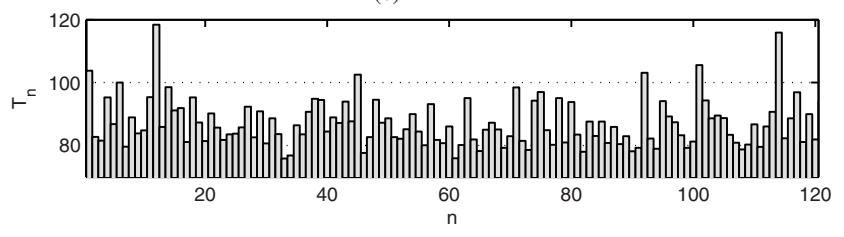

FIG. 3. (Color online) A spatiotemporal code generated by the trajectory where $\omega_{n+1}=\omega_{n}$ for $n=1, \ldots, 5$ and $\eta=10^{-7}$. Panel (a) represents the trajectory being converted into a spatio-temporal code. Panel (b) shows the state sequence $C_{n}$ over a set of epochs $n$, where $C_{n}$ is a reference to state code. Panel (c) shows the residence time sequence $T_{n}$ representing the time spent in a given epoch. 
TABLE II. A combinatorial representation of the two possible period 6 cycles that the system will collapse to given uniform detuning (7), $\delta \neq 0$ and $\eta=0$.

\begin{tabular}{cccccccccccccc}
\hline \hline Epoch & $j_{1}^{k}$ & $j_{2}^{k}$ & $j_{3}^{k}$ & $j_{4}^{k}$ & $j_{5}^{k}$ & $C_{n}$ & Epoch & $j_{1}^{k}$ & $j_{2}^{k}$ & $j_{3}^{k}$ & $j_{4}^{k}$ & $j_{5}^{k}$ & $C_{n}$ \\
\hline$n$ & 3 & 1 & 1 & 3 & 2 & 18 & $n$ & 3 & 1 & 3 & 1 & 2 & 15 \\
$n+1$ & 1 & 3 & 3 & 2 & 1 & 7 & $n+1$ & 1 & 3 & 2 & 3 & 1 & 6 \\
$n+2$ & 3 & 1 & 2 & 1 & 3 & 5 & $n+2$ & 3 & 1 & 1 & 2 & 3 & 8 \\
$n+3$ & 1 & 3 & 1 & 3 & 2 & 16 & $n+3$ & 1 & 3 & 3 & 1 & 2 & 17 \\
$n+4$ & 3 & 1 & 3 & 2 & 1 & 10 & $n+4$ & 3 & 1 & 2 & 3 & 1 & 3 \\
$n+5$ & 1 & 3 & 2 & 1 & 3 & 4 & $n+5$ & 1 & 3 & 1 & 2 & 3 & 9 \\
\hline \hline
\end{tabular}

\section{B. Numerical simulations}

Consider a sequence of residence times for a given uniform detuning with an amplitude of detuning $\delta$. From long numerical simulations using high resolution data integrated using a fourth order Runge-Kutta method followed by an Euler step for the noise, it can be seen that the residence time at each state is close to one of four discrete values; $T_{n}$ $\in\left\{\alpha_{1}, \alpha_{2}, \alpha_{3}, \alpha_{4}\right\}$, where $\alpha_{1}<\alpha_{2}<\alpha_{3}<\alpha_{4}$. More specifically, after an initial transient period of $t$ duration 1000, $T_{n}$ repeats every six epochs, but takes only four different values (see Fig. 4).

Consider now the values of $\left\{T_{n}\right\}$ for a range of values of $\delta$, after the system has passed through the transient period. For each $\delta$ we see the same two possible coding sequences for $C_{n}$ dependent on initial conditions. There is a logarithmic relationship between $\delta$ and the residence times as shown in Fig. 5.

\section{Inverse problem-determining the detuning from a spatiotemporal code}

For computational purposes it is necessary to understand the process by which we could determine the input to a system given a length of spatiotemporal code. We will consider the spatial and temporal coding sequence separately.
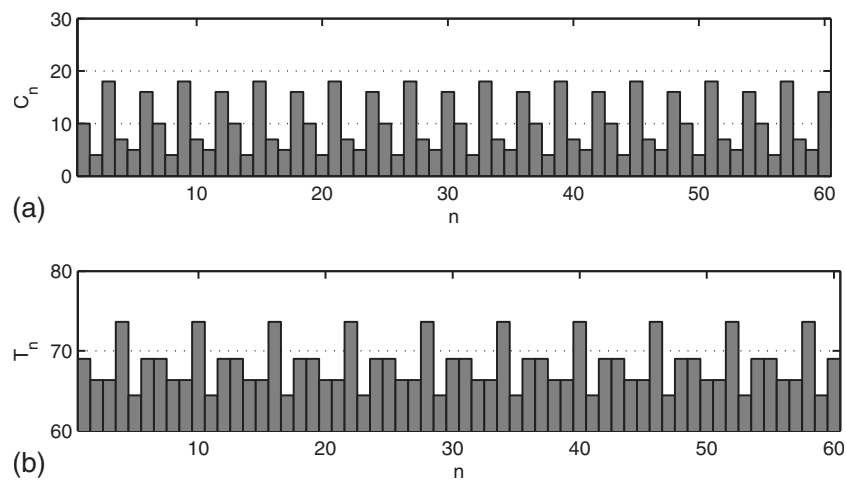

FIG. 4. The spatiotemporal code $\left(C_{n}, T_{n}\right)$, as a function of epoch number $n$, from a numerical simulation with uniform detuning (7) for $\delta=10^{-7}$ and $\eta=0$ after an initial transient period has been allowed to decay. The data shows one of the two period 6 cycles shown in Table II. Notice that while the system passes through six different states, there are only four possible values for the residence times.

\section{Decoding the spatial coding}

As shown in Sec. III B, a uniform detuning will always result in a period 6 cycle in the spatial coding sequence. For the given state in the spatial coding $C_{n}$, consider the oscillators $p, q$ in the unstable cluster such that $j_{p}^{n}=j_{q}^{n}=3$. Note then, that $C_{n+1}$ is equal to either $O_{p}\left(C_{n}\right)$ or $O_{q}\left(C_{n}\right)$. These two cases can be used to compare $\omega_{p}$ and $\omega_{q}$ as follows:

$$
\begin{aligned}
& O_{p}\left(C_{n}\right)=C_{n+1} \Leftrightarrow \omega_{p}>\omega_{q}, \\
& O_{q}\left(C_{n}\right)=C_{n+1} \Leftrightarrow \omega_{q}>\omega_{p} .
\end{aligned}
$$

Given a number of transitions, every oscillator that arrives in an unstable cluster will be subject to a comparison. Consider a graph where each node represents an oscillator and edges between those nodes represent a comparison between those oscillators (both oscillators are in the unstable cluster at the time of a transition). This graph, shown in Fig. 6(a), is bipartite and has a minimum spanning tree of length 4 [see Fig. 6(b)]. This shows that we need to examine only four transitions to determine an ordering in which we can state that two oscillators form the "slow" group and three oscillators form the "fast" group. We call this the slow-fast partition and use this to order $\omega_{i}$ as follows:

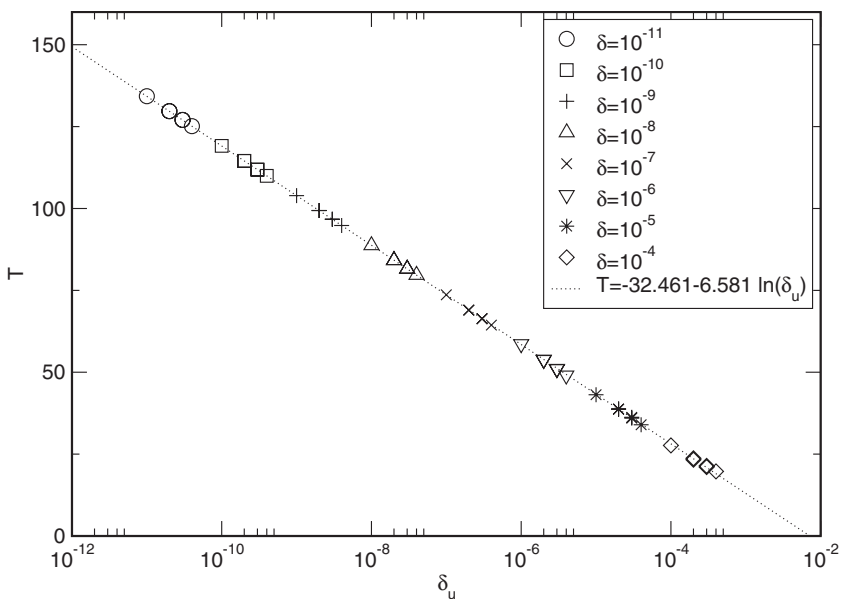

FIG. 5. A plot showing residence time $T$ against $\delta_{u}$. Slope fits to $y=A+B \ln (x)$, where $A=-32.461$ and $B=-6.581 \ln (x)$. 


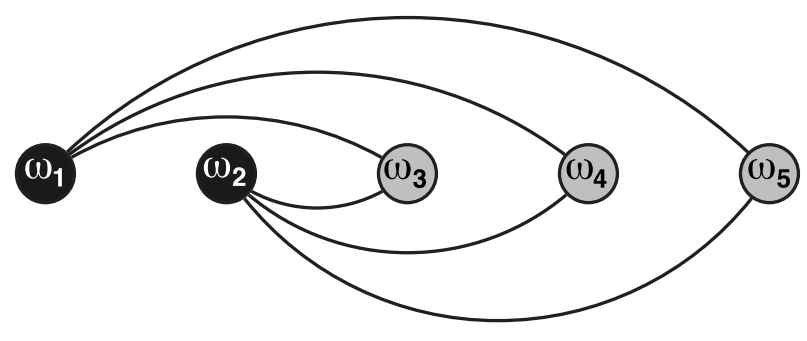

(a)

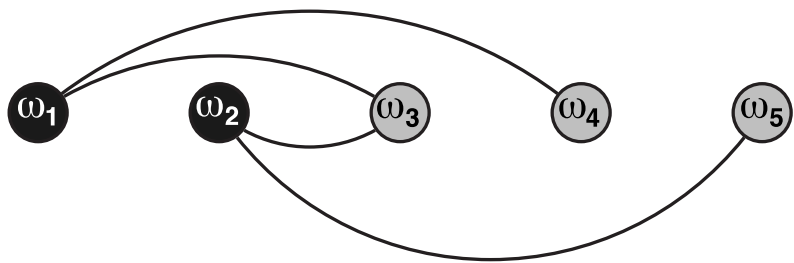

(b)

FIG. 6. For uniform detuning, $\omega_{1}<\omega_{2}<\omega_{3}<\omega_{4}<\omega_{5}$, the dynamics will drive the system into the sequence of states in Table II. This means that only the comparisons shown by lines in graph (a) will be made between oscillator frequencies. Note that at each epoch there is a comparison between one of the two slow oscillators (black) with one of the three fast oscillators (gray) in the slow-fast partition $\{\{1,2\},\{3,4,5\}\}$. Graph (b) shows a minimum spanning tree for (a) which has four edges, meaning we only need to examine four transitions to determine the ordering of the $\omega_{i}$.

$$
\max \left(\omega_{a}, \omega_{b}\right)<\min \left(\omega_{c}, \omega_{d}, \omega_{e}\right),
$$

where $a, b$ are the indices relating to the two oscillators with the smallest amount of detuning and $c, d, e$ are the indices of the oscillators with the largest amount of detuning. Consider the following example. By comparing the 3's from the left period 6 cycle from Table II, the oscillator with the greatest detuning will move to the singleton group (becomes 2), and the oscillator with the least detuning will move into the stable cluster (becomes 1); as such, one can see that $\omega_{1}$ $<\omega_{4}, \omega_{2}<\omega_{3}, \omega_{1}<\omega_{5}, \omega_{2}<\omega_{4}, \omega_{1}<\omega_{3}, \omega_{2}<\omega_{3}$. Hence, $\max \left(\omega_{1}, \omega_{2}\right)<\min \left(\omega_{3}, \omega_{4}, \omega_{5}\right)$.

Without examining the temporal coding, it is not possible to further order the oscillators or make assumptions about the relative amplitudes of detuning.

\section{Decoding the temporal coding}

Given that the amplitudes of detuning for a uniformly detuned input are equidistant, there exist only four possible values for $\delta_{u}$, dependent on the two oscillators being compared in the unstable cluster at any state. The length of any epoch in which oscillators $p, q$ are in the unstable cluster is determined by $\delta_{u}$. If we are near state $s_{i}$ we expect

$$
T_{n}=\tau\left(s_{i}\right),
$$

where $\tau$ is as in Eq. (5). One can then calculate the four possible values of $\delta_{u}$ for a given uniform detuning, which then determines $\delta_{n+1}-\delta_{n}$ for $n=1, \ldots, 4$.

For example, consider the temporal code depicted in Fig. 4. The four distinct average residence times from this simulation are 66.3, 64.4, 69.0, and 73.7. Using 6 we can find the differences between the natural frequencies of the oscillators in the system.

\section{Decoding the spatiotemporal coding}

By considering both the spatial and the temporal coding individually, one can (1) differentiate between the fastest three and the slowest two oscillators, and (2) define the difference in amplitude of detuning between the oscillators (but not which oscillators these differences apply to).

Assume that the system is on one of the period 6 cycles and consider both the residence time $T_{n}$ and the state $C_{n}$ together. By comparing $T_{n}, T_{n+2}$ with the distance between the indices of the oscillators in the unstable cluster (the 3 group) at the states $C_{n}$ and $C_{n+2}$ one can determine the detuning between one of the oscillators $\omega_{a}$ or $\omega_{b}$ and two of the oscillators $\omega_{c}, \omega_{d}$, and $\omega_{e}$.

For example, consider the example in Table II. We know from simulation that the residence times $T_{n}$ for the $C_{n}$ depicted in the table are 66.3, 73.7, 64.4, 69.0, 69.0, and 66.3. Hence, $C_{n}=S_{31132}, C_{n+2}=S_{31213}, T_{n}=66.3$, and $T_{n+2}=64.4$.

$T_{n}>T_{n+2} \Rightarrow \delta_{u}^{n}<\delta_{u}^{n+2} \Rightarrow\left(\omega_{4}-\omega_{1}\right)<\left(\omega_{5}-\omega_{1}\right) \Rightarrow \omega_{4}<\omega_{5}$,

where $\delta_{u}^{n}$ is the value of $\delta_{u}$ at time $n$. By conducting similar comparisons, one can completely order the frequencies $\omega_{1}, \ldots, \omega_{5}$.

\section{Example of nonuniform detuning}

Consider now a spatiotemporal coding as shown in Fig. 7, different from the example shown in Table II. Assuming we know that the detuning input to the system is nonuniform and that the amplitudes of detuning are all different, we consider the process required to decode the information in Fig. 7.

(a)
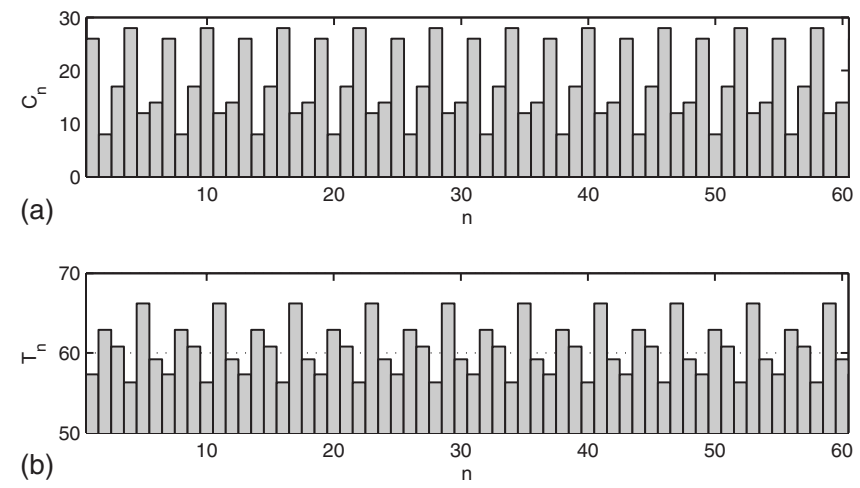

FIG. 7. The spatiotemporal code generated by a simulation with $\eta=0$ and a nonuniform detuning $\left(\omega_{1}, \ldots, \omega_{5}\right)=1.0$ $+(1,10,3,15,6) \sigma$, where $\sigma=10^{-7}$ so $\omega_{1}<\omega_{3}<\omega_{5}<\omega_{2}<\omega_{4}$. Note that the resulting sequence is period 6 , but the residence times are different than those in Fig. 4. 
TABLE III. Decoding the spatiotemporal coding $\left\{C_{n}, T_{n}\right\}$ for a trajectory on the period 6 loop with $\eta$ $=0$. (a) shows an analysis of the data from one of the cycles shown in Table II (b) is from the simulation described in Fig. 7. At each epoch the detuning $\delta_{u}$ between the oscillators in the third column is compared; the switch is determined by which of these has greater frequency. The residence time can be used to estimate the size of $\delta_{u}$ by $\delta_{u} \approx \exp [(32.461+T) /-6.58]$; this is shown in the fourth column. The final column gives the actual detuning; this is approached on taking averages over many visits to the same state.

\begin{tabular}{|c|c|c|c|c|c|}
\hline Epoch & $C_{n}$ & $T_{n}$ & Compares & Inferred $\delta_{u}$ & Actual $\delta_{u}$ \\
\hline \multicolumn{6}{|c|}{ (a) } \\
\hline$n$ & $S_{31132}$ & 66.3 & $\omega_{1}<\omega_{4}$ & $3.03 \times 10^{-7}$ & $3 \times 10^{-7}$ \\
\hline$n+1$ & $S_{13321}$ & 73.7 & $\omega_{2}<\omega_{3}$ & $9.84 \times 10^{-8}$ & $1 \times 10^{-7}$ \\
\hline$n+2$ & $S_{31213}$ & 64.4 & $\omega_{1}<\omega_{5}$ & $4.04 \times 10^{-7}$ & $4 \times 10^{-7}$ \\
\hline$n+3$ & $S_{13132}$ & 69.0 & $\omega_{2}<\omega_{4}$ & $2.01 \times 10^{-7}$ & $2 \times 10^{-7}$ \\
\hline$n+4$ & $S_{31321}$ & 69.0 & $\omega_{1}<\omega_{3}$ & $2.01 \times 10^{-7}$ & $2 \times 10^{-7}$ \\
\hline$n+5$ & $S_{13213}$ & 66.3 & $\begin{array}{l}\omega_{2}<\omega_{5} \\
\text { (b) }\end{array}$ & $3.03 \times 10^{-7}$ & $3 \times 10^{-7}$ \\
\hline$n$ & $S_{12331}$ & 57.3 & $\omega_{3}<\omega_{4}$ & $1.19 \times 10^{-6}$ & $12 \times 10^{-7}$ \\
\hline$n+1$ & $S_{31123}$ & 62.9 & $\omega_{1}<\omega_{5}$ & $5.08 \times 10^{-7}$ & $5 \times 10^{-7}$ \\
\hline$n+2$ & $S_{13312}$ & 60.8 & $\omega_{2}<\omega_{3}$ & $6.99 \times 10^{-7}$ & $7 \times 10^{-7}$ \\
\hline$n+3$ & $S_{32131}$ & 56.3 & $\omega_{1}<\omega_{4}$ & $1.39 \times 10^{-6}$ & $14 \times 10^{-7}$ \\
\hline$n+4$ & $S_{11323}$ & 66.2 & $\omega_{3}<\omega_{5}$ & $3.08 \times 10^{-7}$ & $3 \times 10^{-7}$ \\
\hline$n+5$ & $S_{33112}$ & 59.2 & $\omega_{1}<\omega_{2}$ & $8.92 \times 10^{-7}$ & $9 \times 10^{-7}$ \\
\hline
\end{tabular}

Table III illustrates the process of recreating the input data from a given length of spatiotemporal coding. The values in the columns $C_{n}$ and $T_{n}$ are determined by inspection of the spatiotemporal coding. The fourth column shows which two oscillators are in the unstable cluster during this epoch, and hence, which two oscillators are compared to determine the length of the current epoch and the value of $C_{n}$ for the next epoch. Now estimate the value of $\delta_{u}$ for the compared oscillators by $\delta_{u} \approx \exp [(32.461+T) /-6.58]$. The final column shows the actual value of $\delta_{u}$ for comparison. The estimate of $\delta_{u}$ approaches the value of $\delta_{u}$ on taking averages over many visits to the same state.

\section{DETUNING IN THE PRESENCE OF NOISE}

Adding noise to the system adds a level of uncertainty to the trajectory of the system. We evaluate how robust our model is for $\eta \neq 0$ and investigate how much information about the detuning can be extracted, dependent on the level of noise introduced to the system.

\section{A. Influence of noise on the spatial coding}

Consider the spatial coding sequence $C_{n}$ and the residence time sequence $T_{n}$ for a particular trajectory (Fig. 8). Let $p_{i}$ $\in\left(p_{1}, \ldots, p_{30}\right)$ and $\Sigma_{i=1}^{30} p_{i}=1$ be the relative frequency that, in a given epoch, the system is at state $s_{i}$; i.e.,

$$
p_{i}=\lim _{N \rightarrow \infty} \frac{\mathcal{N}\left\{1 \leqslant n \leqslant N: C_{n}=i\right\}}{N} .
$$

Let $P_{i, j}$ be the relative frequency that the state sequence advances from state $s_{i}$ to $s_{j}$ defined as

$$
P_{i, j}=\lim _{N \rightarrow \infty} \frac{\mathcal{N}\left\{n \leqslant N: c_{n}=i, c_{n+1}=j\right\}}{\mathcal{N}\left\{n \leqslant N: c_{n}=i\right\}} .
$$

Then it can be verified that, for any $i$,

$$
\sum_{j=1}^{30} P_{i, j}=1,
$$

and $P$ can be interpreted as a transition matrix.

Some examples of transition matrices from numerical simulations with constant uniform detuning and increasing noise are depicted in Fig. 9.

We observe that the spatial coding is approximately a Markov chain; the transitions from epoch $n$ to $n+1$ are ap-
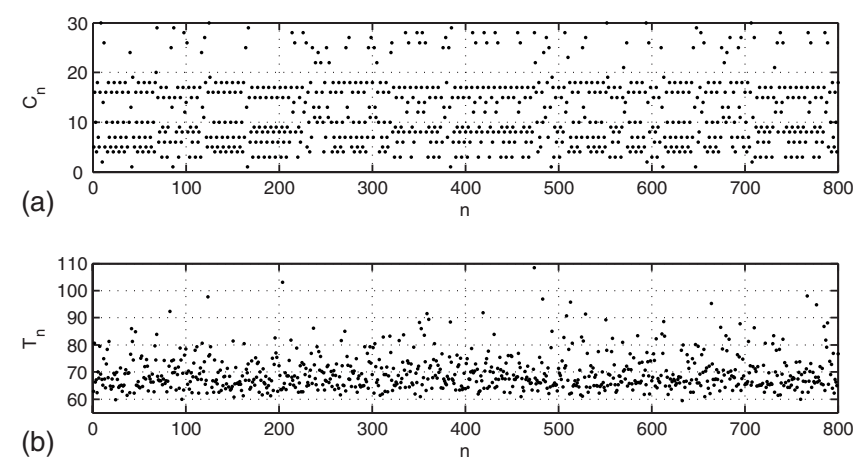

FIG. 8. A spatiotemporal code generated by a numerical simulation over 800 epochs as in Fig. 3 where there is noise present; $\eta / \delta=5.0$. Note there is an apparently random switching between the two possible cycles of length six, plus some occasional longer deviations. This is the activity we see in Fig. 9(c). 
(a) $\eta / \sigma=1.0$

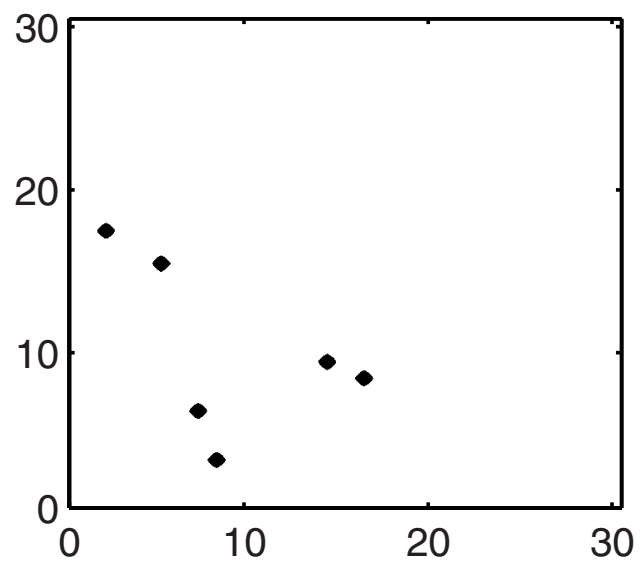

(c) $\eta / \sigma=5.0$

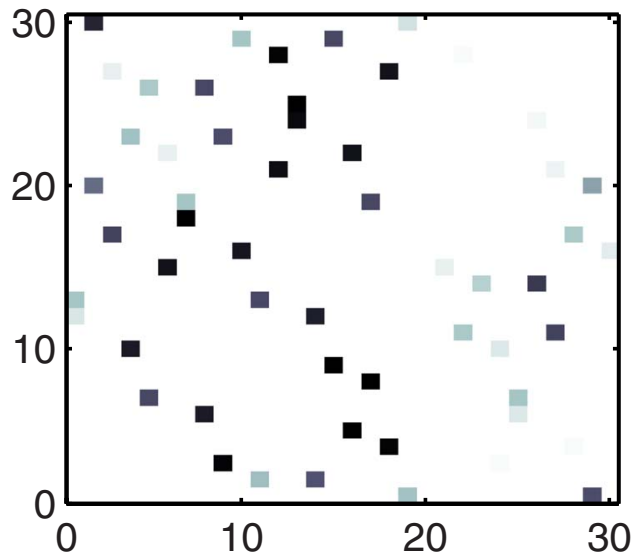

(b) $\eta / \sigma=2.0$

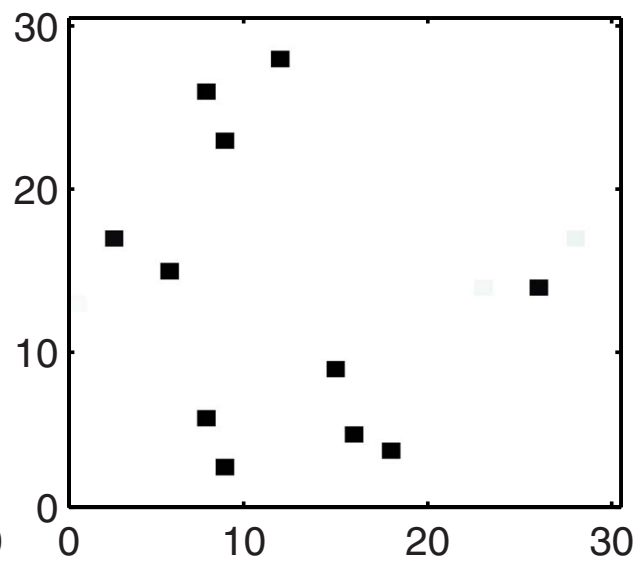

(d) $\eta / \sigma=100.0$

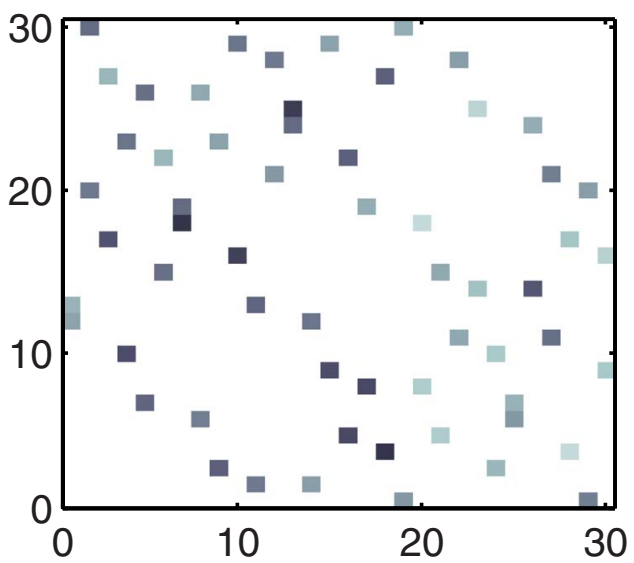

FIG. 9. (Color online) $P_{i, j}$ for long simulations with uniform detuning $\delta=10^{-7}$ and varying noise $\eta$. The horizontal axis represents $i$, and the vertical axis represents $j$. The dark squares indicate a transition probability close to 1 while the white squares indicate a transition probability close to 0 . As before, an initial transient is disregarded. Panel (a) $(\eta / \delta=1.0)$ corresponds to the case where noise apparently has no effect, as seen in Fig. 4. Panel (b) $(\eta / \delta=2.0)$ shows the effect of low noise that is sufficient to occasionally nudge the system between the two stable period 6 cycles present for this detuning. Panel (c) $(\eta / \delta=5.0)$ corresponds to the coding from Fig. 8. Panel $(\mathrm{d})(\eta / \delta$ $=100.0$ ) corresponds to the case where detuning is comparatively weak and all edges are traversed.

parently independent of those for epoch $n-1$ to $n$. The entropy of the spatial coding is given by [13]

$$
\xi=-\sum_{i, j} p_{i} P_{i, j} \ln P_{i, j}
$$

Note $\xi=0$ corresponds to an eventually periodic trajectory, while increasing $\xi$ is associated with an increasing range of possibilities of the future trajectory.

For fixed $\delta=10^{-7}$ and varying $0.01<\frac{\eta}{\delta}<100$, Fig. 10 shows that the entropy for the system increases from zero up to the theoretical maximum for the network in Fig. 2, namely, $0 \leqslant \xi \leqslant \ln 2$. Specifically, the entropy determines the average likelihood that, when the system is at state $C_{n}$, the next state $C_{n+1}$ is not that which is predicted by the ordering of the natural frequencies of the oscillators.

For $\eta / \delta<1.0$ and $\xi=0$ the system will follow the period 6 coding sequence indefinitely. $\eta / \delta=2.0$ corresponds to Fig. 9 (b), where the coding sequence deviates from the period 6 cycle on occasion, but quickly returns to the cycle by a predetermined route. $\eta / \delta=100.0$ corresponds to Fig. 9(d), where the detuning has a minimal effect and the system is almost completely driven by noise. In summary, this shows that the spatial coding of the system is very robust to noise, up to a strength comparable to the order of the amplitude of detuning.

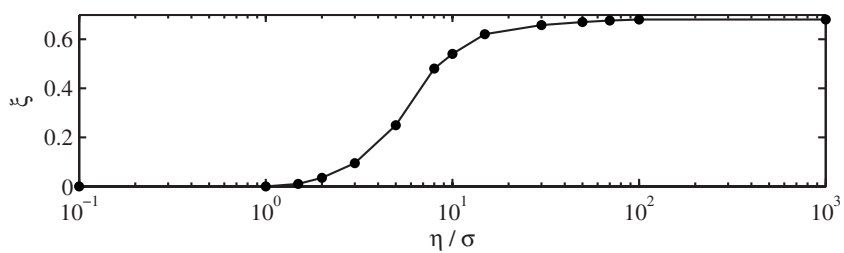

FIG. 10. The entropy $\xi$ in the spatial coding of the system as a function of $\eta / \delta$ from numerical simulations. At $\eta / \delta<0.5$, $\xi \approx 0$ - there is little uncertainty in the path that the system will follow. The entropy $\xi$ increases quickly between $0.5<\eta / \delta<10.0$, where it is close to the theoretical entropy of an unbiased random walk on Fig. 2, namely, $\xi=\ln (2)$. 


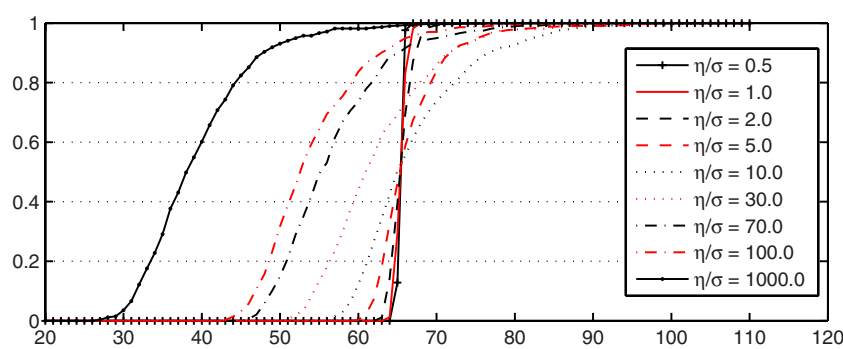

FIG. 11. (Color online) Each data set shows the cumulative frequency $f(T)$ of $n$ such that $C_{n}=18$ and $T_{n}<T$ from the simulations depicted in Fig. 10, more precisely $f(T) \mathcal{N}\left\{n: C_{n}=18\right.$ and $T_{n}$ $\leqslant T\} / \mathcal{N}\left\{n: C_{n}=18\right\}$. As $\eta / \delta$ increases the variance in the sequence of residence times $T_{n}$ increases and the mean epoch length decreases.

\section{B. Influence of noise on the temporal coding}

For $\eta=0$ and $\delta>0$ we expect the sequence $T_{n}$ to be dependent on the detuning $\delta_{u}$ of the unstable cluster for state $C_{n}$, given by Eq. (5). Long simulations were run for a number of different $\eta / \delta$ and the distribution of $T_{n}$ obtained is shown in Fig. 11. From this figure we see that as $\eta / \delta$ increases, the variance of $T_{n}$ increases, while the mean decreases slowly for $\eta / \delta<10$ and more rapidly for $\eta / \delta>10$ (for comparison, see [11]).

Examining the mean and variance of the distribution of residence times $T_{n}$ shows that the temporal coding of the system is also robust to noise up to $\eta / \delta \approx 10$. In fact, the mean of the distribution does not significantly change even when the strength of the noise is of the same order as the amplitude of detuning.

\section{DISCUSSION}

This paper demonstrates that the itinerary between cluster states for a robust heteroclinic cycle for the system of five coupled phase oscillators (1) can be used to encode all information about the "inputs" to the system in terms of the detunings between oscillators. Examining the itinerary (spatial coding) and the residence times at each of these states (temporal coding) gives enough information to obtain all frequency differences between oscillators. The spatial coding gives a partition of the oscillators into two slow and three fast oscillators, while the average residence times allow one to estimate the frequency differences. At each cluster state only two of the oscillators are in the unstable cluster. This enables one to estimate the frequency difference as an initial perturbation that grows exponentially. It is quite surprising that there is not more information loss; for example, if the coupling were such that an in-phase solution were stable, the response to very low amplitude inputs would be linear and very small, unlike the situation here, where very low amplitude inputs result in a large response.

For larger populations of phase oscillators, very similar behavior can be observed except that the coding becomes much more difficult to describe in detail owing to a combinatorial explosion in the number of cluster states potentially involved, the larger number of switching possibilities involved, and the unstable manifold of each cluster state being higher dimensional. For instance, using the same coupling as in Eq. (2) for $N=9$ oscillators one can find a robust attracting heteroclinic cycle involving over 600 cluster states joined by four-dimensional manifolds [14]. In this case the dynamics in the presence of detuning does not compare all oscillators; there is, as in the case $N=5$, also an eventually period 6 itinerary but this compares only the fastest five oscillators. Nonetheless, we conjecture that there are other coupling functions for higher $N$ cases that will robustly and fully encode the phase differences by involving other cluster states.

We have illustrated, by computation of the entropy of the resulting spatiotemporal sequence, that the effect of noise on the spatiotemporal code generation is surprisingly small up to quite high amplitude noise. In particular, the entropy of the spatial coding remains almost zero up to a signal to noise ratio of 10:1.

Winnerless competition, where an attractor is a heteroclinic network, has been used to describe a variety of neural systems, most particularly in rate-based competition models $[5,15]$. It is particularly useful for describing the dynamics of neural processes that lead to sequence generation and/or spatiotemporal code generation. The model considered here can be considered as an example of winnerless competition that is not a rate model. In our model, cluster states play the same role as subspaces for rate models where one or more of the neural units has a zero rate. It illustrates that winnerless competition can occur in phase oscillator models and, at least for small numbers of oscillators, that the spatiotemporal coding is efficient and invertible. We suggest that these models can give useful insight to the dynamics of neural assemblies where once again, "instability makes sense" [16].
[1] F. C. Hoppensteadt and E. M. Izhikevich, Weakly Connected Neural Networks, Applied Mathematical Sciences Vol. 126 (Springer-Verlag, Berlin, 1997).

[2] D. Hansel, G. Mato, and C. Meunier, Phys. Rev. E 48, 3470 (1993).

[3] H. Kori and Y. Kuramoto, Phys. Rev. E 63, 046214 (2001).

[4] M. Rabinovich, A. Volkovskii, P. Lecanda, R. Huerta, H. D. I. Abarbanel, and G. Laurent, Phys. Rev. Lett. 87, 068102 (2001).
[5] M. Rabinovich, R. Huerta, and G. Laurent, Science 321, 48 (2008)

[6] P. Ashwin, G. Orosz, J. Wordsworth, and S. Townley, SIAM J. Appl. Dyn. Syst. 6, 728 (2007).

[7] P. Ashwin and J. Borresen, Phys. Rev. E 70, 026203 (2004).

[8] P. Ashwin, O. Burylko, and Y. Maistrenko, Physica D 237, 454 (2008).

[9] P. Ashwin, G. Orosz, and J. Borresen, University of Exeter (unpublished). 
[10] Linearizing our proposed system about an arbitrary cluster state allows one to investigate the stability of that state. We find the following eigenvalues: $\lambda_{1}=0, \lambda_{2}<0 \in \mathbb{R}, \lambda_{3}>0 \in \mathbb{R}$, $\lambda_{4}=x+i y$, and $\lambda_{5}=\lambda_{4}^{*}=x-i y$ for $x<0$. The corresponding eigenvectors $v_{1}, \ldots, v_{5}$ provide interesting information about the dynamics near a given cluster state [6]. $v_{1}$ represents translation along the $S^{1}$ group orbit of the system; $v_{2}$ and $v_{3}$ represent splitting the oscillators in the groups labeled by 1 's and 3 's, respectively; $v_{4}$ and $v_{5}$ correspond to motion in the subspace around the cluster state and represent perturbations that do not split the clusters.
[11] E. Stone and P. Holmes, SIAM J. Appl. Math. 50, 726 (1990).

[12] There are other sensible ways to identify the cluster state; for example, one can choose a threshold $\xi>0$ and identify that phases $\theta$ are near the state $S_{11332}$ if $\left|\theta_{1}-\theta_{2}\right|<\xi,\left|\theta_{3}-\theta_{4}\right|<\xi$, $\left|\theta_{1}-\theta_{4}\right|>\xi$, and $\left|\theta_{5}-\theta_{j}\right|>\xi$ for all $j \neq 5$.

[13] P. Walters, An Introduction to Ergodic Theory, Graduate Texts in Mathematics Vol. 79 (Springer-Verlag, Berlin, 1982).

[14] G. Orosz, P. Ashwin, and S. Townley (unpublished).

[15] M. Rabinovich, P. Varona, A. I. Selverston, and H. D. I. Abarbanel, Rev. Mod. Phys. 78, 1213 (2006).

[16] P. Ashwin and M. Timme, Nature (London) 436, 36 (2005). 